\title{
HYPERBOLIC MANNING-ROSEN POTENTIAL UNDER SPIN AND PSEUDOSPIN SYMMETRIES OF THE DIRAC EQUATION WITH COULOMB-HULTHÉN-LIKE TENSOR INTERACTIONS
}

\author{
Akpan N. Ikot ${ }^{1 *}$, E. Maghsoodi ${ }^{2}$, S. Zarrinkamar ${ }^{3}$, H. Hassanabadi ${ }^{2}$ \\ ${ }^{1}$ Department of Physics, University of Port Harcourt, Choba P. M. B. 5323, Port Harcourt, Nigeria \\ ${ }^{2}$ Department of Basic Sciences, Shahrood Branch, Islamic Azad University, Shahrood, Iran \\ ${ }^{3}$ Department of Basic Sciences, Garmsar Branch, Islamic Azad University, Garmsar, Iran \\ *ndemikotphysics@gmail.com
}

(Received October 01, 2013; received in final form April 04, 2014)

\begin{abstract}
The Dirac equation with the hyperbolic Manning-Rosen potential including the combined Coulomb-like and Hulthén-type tensor interactions is investigated. The approximate analytical bound state solutions of the Dirac equation with the hyperbolic Manning-Rosen potential, energy equations and the corresponding unnormalized wave functions are obtained in a closed form using the Nikiforov-Uvarov method. We have also reported the numerical results to show the effect of tensor interaction.
\end{abstract}

Key words: hyperbolic Manning-Rosen potential, tensor interactions Nikiforov-Uvarov method, Spin and pseudospin symmetries.

PACS number(s): 03.65.Ge, 03.65.Pm, 03.65.Db

\section{INTRODUCTION}

The Manning-Rosen potential [1, 2] is an important diatomic molecular potential model. Recently a number of researchers have worked on the actual form of this potential [3-7]. For instance, Taskın solved approximately the Dirac equation with the form Manning-Rosen actually potential in Ref. [4]. Wei and Dong applied a Pekeristype approximation to the pseudo-centrifugal term the pseudospin symmetry of a Dirac nucleon subjected to scalar and vector Manning-Rosen potentials including the spin-orbit coupling term in Ref. [5]. Chen et al. in Ref. [7] solved approximately the Dirac equation with the Manning-Rosen potential and calculated the bound state energy eigenvalues and the associated two-component spinors of the Dirac particle.

A number of other closely related potentials are also of some interest though most often one is simply revisiting the Eckart potential in disguise. Note that the Manning-Rosen potential can be obtained from the Eckart potential. In particular, Manning-Rosen can be written the Eckart potential by appropriately choosing the parameters. The search for the solutions of Dirac equation with physically motivated potential models will lead to the discovery of a new phenomena in addition to the spin and pseudospin symmetry discovered many years ago in the nuclei of atom in the Dirac theory [810], in nuclear physics to explain the an experimental observation of quasi-degeneracy in single-nucleon doublets between normal parity orbitals $\left(n, l, j=l+\frac{1}{2}\right)$ and $\left(n-1, l+2, j=l+\frac{3}{2}\right)$, where $n, l$ and $j$ represent the radial, orbital and total angular momentum quantum numbers, respectively. These symmetries have been used to form an effective nuclear shell model [11] and to elucidate other different physical phenomena in the nuclear structure such as deformation, super-deformation, magnetic moment and identical bands [12]. Pseudospin and spin symmetries concept on a number of potentials have been investigated by many researchers in the field. These potentials include Woods-Saxon [13], ManningRosen [1], Eckart potential [14], harmonic oscillator [15], Hyllerass potential [16], Deng-Fan [17] and others [18]. The spin and pseudospin symmetries under various phenomenological potentials have been investigated using various methods such as asymptotic iteration method (AIM) [19], Nikiforov-Uvarov (NU) method [20], supersymmetric quantum mechanics (SUSYQM) [21], and others [22]. The spin and pseudospin symmetries of Dirac equation have a significant role in nuclear and harmonic spectroscopy [23-26]. In this formulation, the tensor interaction has attracted a great attention as it removes degeneracy between the doublets [27]. In most studies, due to the mathematical structure of the problem, the tensor interaction is considered as Coulomb-like [28] or Cornell interaction. Hassanabadi et al. introduced the Yukawa tensor interaction [29] in the Dirac theory. Recently, Ikot et al. [30] introduced the generalized tensor interaction in the Dirac theory and as a matter of fact removed degeneracy from the spin and pseudospin doublet as should be expected.

The Manning-Rosen potential has been one of the most useful and elegant potential models for studying energy eigenvalues of diatomic molecules [31]. As an empirical potential, the Manning-Rosen potential gives an excellent description of the interaction between two atoms in a diatomic molecule, and it is very good for describing such interactions close to the surface [32].

In the present paper, we consider hyperbolic ManningRosen potentials including a novel Coulomb-Hulthén like tensor interaction terms and proceed on an approximate analytical manner. In the next section, we will introduce the Nikiforov-Uvarov (NU) technique we will use to solve our obtained differential equation. 


\section{THE NIKIFOROV-UVAROV METHOD}

The NU method can solve a second-order differential equation of the form [20]

$$
\psi_{n}^{\prime \prime}(s)+\frac{\tilde{\tau}(s)}{\sigma(s)} \psi_{n}^{\prime}(s)+\frac{\tilde{\sigma}(s)}{\sigma^{2}(s)} \psi_{n}(s)=0,
$$

where $\sigma(s)$ and $\tilde{\sigma}(s)$ are polynomials, at most the second degree, and $\tilde{\tau}(s)$ is a first-degree polynomial. To make the application of the NU method simpler and more direct, we introduce a more compact presentation of the idea. In order to do this, we rewrite Eq. (1) as follows [32]

$$
\begin{aligned}
& \psi_{n}^{\prime \prime}(s)+\left(\frac{c_{1}-c_{2} s}{s\left(1-c_{3} s\right)}\right) \psi_{n}^{\prime}(s) \\
& +\left(\frac{-\xi_{1} s^{2}+\xi_{2} s-\xi_{3}}{s^{2}\left(1-c_{3} s\right)^{2}}\right) \psi_{n}(s)=0,
\end{aligned}
$$

in which

$$
\psi_{n}(s)=\phi(s) y_{n}(s) .
$$

Comparing Eq. (1) with Eq. (2), we obtain the following identifications:

$$
\begin{aligned}
& \tilde{\tau}(s)=c_{1}-c_{2} s, \\
& \sigma(s)=s\left(1-c_{3} s\right), \\
& \tilde{\sigma}(s)=-\xi_{1} s^{2}+\xi_{2} s-\xi_{3},
\end{aligned}
$$

Using the NU method [27, 28], we obtain the following required parameters:

(I) the relevant constant:

$$
\begin{aligned}
c_{4} & =\frac{1}{2}\left(1-c_{1}\right), & c_{5} & =\frac{1}{2}\left(c_{2}-2 c_{3}\right), \\
c_{6} & =c_{5}^{2}+\xi_{1}, & c_{7} & =2 c_{4} c_{5}-\xi_{2}, \\
c_{8} & =c_{4}^{2}+\xi_{3}, & c_{9} & =c_{3} c_{7}+c_{3}^{2} c_{8}+c_{6} . \\
c_{10} & =c_{1}+2 c_{4}+2 \sqrt{c_{8}}, & c_{11} & =c_{2}-2 c_{5}+2\left(\sqrt{c_{9}}+c_{3} \sqrt{c_{8}}\right) \\
c_{12} & =c_{4}+\sqrt{c_{8}}, & c_{13} & =c_{5}-\left(\sqrt{c_{9}}+c_{3} \sqrt{c_{8}}\right)
\end{aligned}
$$

(II) the essential polynomial functions:

$$
\begin{aligned}
& \pi(s)=c_{4}+c_{5} s-\left[\left(\sqrt{c_{9}}+c_{3} \sqrt{c_{8}}\right) s-\sqrt{c_{8}}\right] \\
& k=-\left(c_{7}+2 c_{3} c_{8}\right)-2 \sqrt{c_{8} c_{9}} \\
& \tau(s)=c_{1}+2 c_{4}-\left(c_{2}-2 c_{5}\right) s \\
& \quad-2\left[\left(\sqrt{c_{9}}+c_{3} \sqrt{c_{8}}\right) s-\sqrt{c_{8}}\right] \\
& \tau^{\prime}(s)=-2 c_{3}-2\left(\sqrt{c_{9}}+c_{3} \sqrt{c_{8}}\right)<0 .
\end{aligned}
$$

(III) The energy equation:

$$
\begin{aligned}
& c_{2} n-(2 n+1) c_{5}+(2 n+1)\left(\sqrt{c_{9}}+c_{3} \sqrt{c_{8}}\right) \\
& +n(n-1) c_{3}+c_{7}+2 c_{3} c_{8}+2 \sqrt{c_{8} c_{9}}=0 .
\end{aligned}
$$

(IV) The wave functions

$$
\begin{gathered}
\rho(s)=s^{c_{10}}\left(1-c_{3} s\right)^{c_{11}}, \\
\phi(s)=s^{c_{12}}\left(1-c_{3} s\right)^{c_{13}}, \quad c_{12}>0, \quad c_{13}>0,
\end{gathered}
$$

$$
\begin{gathered}
y_{n}(s)=P_{n}^{\left(c_{10}, c_{11}\right)}\left(1-2 c_{3} s\right), \quad c_{10}>-1, c_{11}>-1 \\
\psi_{n \kappa}(s)=N_{n \kappa} s^{c_{12}}\left(1-c_{3} s\right)^{-c_{12}-\frac{c_{13}}{c_{3}}} \\
\times P_{n}^{\left(c_{10}-1, \frac{c_{11}}{c_{3}}-c_{10}-1\right)}\left(1-2 c_{3} s\right)
\end{gathered}
$$

where $P_{n}^{(\mu, \nu)}(x), \mu>-1, \nu>-1$, and $x \in[-1,1]$ are Jacobi polynomials with

$$
P_{n}^{(\alpha, \beta)}(1-2 s)=\frac{(\alpha+1)_{n}}{n !}{ }_{2} F_{1}(-n, 1+\alpha+\beta+n ; \alpha+1 ; s),
$$

and $N_{n \kappa}$ is a normalization constant. Where $c_{12}>0$, $c_{13}>0$ and $s \in\left[0,1 / c_{3}\right], c_{3} \neq 0$. This method has been extensively used to solve various second-order differential equations in quantum mechanics such as Schredinger, Klein-Gordon, Duffin-Kemmer-Petiau (DKP), spinlessSalpeter and Dirac equations [16-18].

\section{THEORY OF DIRAC EQUATION}

The Dirac equation for spin- $\frac{1}{2}$ particles moving in an attractive scalar potential $S(r)$, a repulsive vector potential $V(r)$ and a tensor potential $U(r)$ in the relativistic unit $(\hbar=c=1)$ is $[8-11]$

$$
\begin{aligned}
& {[\boldsymbol{\alpha} \cdot \boldsymbol{p}+\beta(M+S(r))-i \beta \boldsymbol{\alpha} \cdot \hat{r} U(r)] \psi(r)} \\
& =[E-V(r)] \psi(r),
\end{aligned}
$$

where $E$ is the relativistic energy of the system, $\boldsymbol{p}=-i \boldsymbol{\nabla}$ is the three dimensional momentum operator and $M$ is the mass of the fermionic particle. $\alpha, \beta$ are the $4 \times 4$ Dirac matrices given as

$$
\boldsymbol{\alpha}=\left(\begin{array}{cc}
0 & \boldsymbol{\sigma}_{i} \\
\boldsymbol{\sigma}_{i} & 0
\end{array}\right), \quad \beta=\left(\begin{array}{cc}
I & 0 \\
0 & -I
\end{array}\right)
$$

where $I$ is a $2 \times 2$ unit matrix and $\boldsymbol{\sigma}_{i}$ are the Pauli threevector matrices:

$$
\sigma_{1}=\left(\begin{array}{cc}
0 & 1 \\
1 & 0
\end{array}\right), \sigma_{2}=\left(\begin{array}{cc}
0 & -i \\
i & 0
\end{array}\right), \sigma_{3}=\left(\begin{array}{cc}
1 & 0 \\
0 & -1
\end{array}\right) .
$$

The eigenvalues of the spin-orbit coupling operator are $\kappa=\left(j+\frac{1}{2}\right)>0, \kappa=-\left(j+\frac{1}{2}\right)<0$ for the unaligned $j=l-\frac{1}{2}$ and aligned spin $j=l+\frac{1}{2}$, respectively. The set $\left(H, K, J^{2}, J_{z}\right)$ forms a complete set of conserved quantities. Thus, we can write the spinors as [8],

$$
\psi_{n \kappa}(r)=\frac{1}{r}\left(\begin{array}{l}
F_{n \kappa}(r) Y_{j m}^{l}(\theta, \varphi) \\
i G_{n \kappa}(r) Y_{j m}^{\tilde{l}}(\theta, \varphi)
\end{array}\right)
$$

where $F_{n \kappa}(r), G_{n \kappa}(r)$ represent the upper and lower components of the Dirac spinors and $\tilde{\ell}$ is pseudo-orbital angular momentum, which is defined as $\tilde{\ell}=\ell+1$ for the aligned $\operatorname{spin} j=\tilde{\ell}-1 / 2$ and $\tilde{\ell}=\ell-1$ for the unaligned spin $j=\tilde{\ell}+1 / 2 . Y_{j m}^{l}(\theta, \varphi), Y_{j m}^{\tilde{l}}(\theta, \varphi)$ are the spin and 
pseudospin spherical harmonics and $m$ is the projection on the $z$-axis. Using well-known identities [10],

$$
\begin{aligned}
& (\boldsymbol{\sigma} \cdot \boldsymbol{A})(\boldsymbol{\sigma} \cdot \boldsymbol{B})=\boldsymbol{A} \cdot \boldsymbol{B}+i \boldsymbol{\sigma} \cdot(\boldsymbol{A} \times \boldsymbol{B}), \\
& \boldsymbol{\sigma} \cdot \boldsymbol{p}=\boldsymbol{\sigma} \cdot \hat{r}\left(\hat{r} \cdot \boldsymbol{p}+i \frac{\boldsymbol{\sigma} \cdot \boldsymbol{L}}{r}\right)
\end{aligned}
$$

as well as the relations

$$
\begin{aligned}
& (\boldsymbol{\sigma} \cdot \boldsymbol{L}) Y_{j m}^{\tilde{l}}(\theta, \varphi)=(\kappa-1) Y_{j m}^{\tilde{l}}(\theta, \varphi) \\
& (\boldsymbol{\sigma} \cdot \boldsymbol{L}) Y_{j m}^{l}(\theta, \varphi)=-(\kappa+1) Y_{j m}^{l}(\theta, \varphi) \\
& (\boldsymbol{\sigma} \cdot \hat{r}) Y_{j m}^{l}(\theta, \varphi)=-Y_{j m}^{\tilde{l}}(\theta, \varphi) \\
& (\boldsymbol{\sigma} \cdot \hat{r}) Y_{j m}^{\tilde{l}}(\theta, \varphi)=-Y_{j m}^{l}(\theta, \varphi)
\end{aligned}
$$

we find the following two coupled first-order Dirac equation [8-11],

$$
\begin{aligned}
& \left(\frac{d}{d r}+\frac{\kappa}{r}-U(r)\right) F_{n \kappa}(r)=\left(M+E_{n \kappa}-\Delta(r)\right) G_{n \kappa}(r), \\
& \left(\frac{d}{d r}-\frac{\kappa}{r}+U(r)\right) G_{n \kappa}(r)=\left(M-E_{n \kappa}+\Sigma(r)\right) F_{n \kappa}(r),
\end{aligned}
$$

where

$$
\begin{aligned}
& \Delta(r)=V(r)-S(r) \\
& \Sigma(r)=V(r)+S(r)
\end{aligned}
$$

Eliminating $F_{n \kappa}(r)$ and $G_{n \kappa}(r)$ in Eqs. (22) and (23), we obtain the second-order Schrödinger-like equation

$$
\left.\begin{array}{l}
\left\{\begin{array}{l}
\frac{d^{2}}{d r^{2}}-\frac{\kappa(\kappa+1)}{r^{2}}+\frac{2 \kappa U(r)}{r}-\frac{d U(r)}{d r}-U^{2}(r)-\left(M+E_{n \kappa}-\Delta(r)\right)\left(M-E_{n \kappa}+\Sigma(r)\right) \\
+\frac{\frac{d \Delta(r)}{d r}\left(\frac{d}{d r}+\frac{\kappa}{r}-U(r)\right)}{\left(M+E_{n \kappa}-\Delta(r)\right)}
\end{array}\right\} F_{n \kappa}(r)=0 \\
\left\{\begin{array}{l}
\frac{d^{2}}{d r^{2}}-\frac{\kappa(\kappa-1)}{r^{2}}+\frac{2 \kappa U(r)}{r}+\frac{d U(r)}{d r}-U^{2}(r)-\left(M+E_{n \kappa}-\Delta(r)\right)\left(M-E_{n \kappa}+\Sigma(r)\right) \\
-\frac{d \Sigma(r)}{d r}\left(\frac{d}{d r}-\frac{\kappa}{r}+U(r)\right)
\end{array}\right\} G_{n \kappa}(r)=0
\end{array}\right\}
$$

where $\kappa(\kappa-1)=\tilde{l}(\tilde{l}+1), \kappa(\kappa+1)=l(l+1)$.

\section{PSEUDOSPIN AND SPIN SYMMETRY LIMITS UNDER COULOMB-LIKE TENSOR INTERACTION}

In this section, we intend to investigate the Dirac equation with Manning-Rosen potential in the presence of Coulomb-Hulthén tensor interactions.

\section{A. Pseudospin symmetry in the Dirac equation with Coulomb-Hulthén-like tensor interaction}

The pseudospin symmetry occurs in the Dirac equation when $\frac{d \Sigma(r)}{d r}=0$ or equivalently $\Sigma(r)=C_{\mathrm{ps}}=$ const [8-11]. To investigate the approximate analytical solution of the Manning-Rosen potential, we consider the sum of the scalar and vector potential as [33-34],

$$
\begin{aligned}
\Delta(r) & =\alpha_{r} \frac{\cosh ^{2}\left(\frac{r}{2 b}\right)}{\sinh ^{2}\left(\frac{r}{2 b}\right)}+\beta_{r} \frac{\cosh \left(\frac{r}{2 b}\right)}{\sinh \left(\frac{r}{2 b}\right)}+\gamma_{r} \\
& =\alpha_{r}\left(\frac{1+e^{-\delta r}}{1-e^{-\delta r}}\right)^{2}+\beta_{r}\left(\frac{1+e^{-\delta r}}{1-e^{-\delta r}}\right)+\gamma_{r},
\end{aligned}
$$

$$
\begin{aligned}
\delta & =\frac{1}{b}, \quad \alpha_{r}=\frac{\alpha(\alpha-1)}{4 b^{2}}, \\
\beta_{r} & =\frac{1}{2 b^{2}}[\alpha(\alpha-1)-A], \\
\gamma_{r} & =\alpha_{r}+\frac{A}{2 b^{2}},
\end{aligned}
$$

where the parameters $\alpha, A$ and $b$ are independent potential parameters and in addition to our newly proposed novel Coulomb-Hulthén tensor interactions term defined as,

$$
U(r)=-\left(\frac{H_{c}}{r}+V_{\mathrm{H}} \frac{e^{-\delta r}}{1-e^{-\delta r}}\right) \quad r \geq R_{c}
$$

with

$$
H_{c}=\frac{z_{a} z_{b} e^{2}}{4 \pi \varepsilon_{0}},
$$

where $R_{c}$ is the Coulomb radius, $z_{a}$ and $z_{b}$ denote the charges of the projectile and target nuclei $\mathrm{b}$ and $V_{\mathrm{H}}$ is the Hulthén parameter respectively $[35,36]$. 
Substituting the above equations into Eq. (27) yields

$$
\begin{aligned}
& \left\{\frac{d^{2}}{d r^{2}}-\frac{\kappa(\kappa-1)}{r^{2}}-\frac{2 \kappa H_{c}}{r^{2}}-\frac{H_{c}^{2}}{r^{2}}+\frac{H_{c}}{r^{2}}-\left(M+E_{n, \kappa}^{\mathrm{ps}}\right)\left(M-E_{n, \kappa}^{\mathrm{ps}}+C_{\mathrm{ps}}\right)-\frac{2 \kappa V_{\mathrm{H}} e^{-\delta r}}{r\left(1-e^{-\delta r}\right)}\right. \\
& -\frac{2 H_{c} V_{\mathrm{H}} e^{-\delta r}}{r\left(1-e^{-\delta r}\right)}-\frac{V_{\mathrm{H}}^{2} e^{-2 \delta r}}{\left(1-e^{-\delta r}\right)^{2}}+\frac{\delta V_{\mathrm{H}} e^{-\delta r}}{\left(1-e^{-\delta r}\right)^{2}}+\left(M-E_{n \kappa}^{\mathrm{ps}}+C_{\mathrm{ps}}\right) \alpha_{r}\left(\frac{1+e^{-\delta r}}{1-e^{-\delta r}}\right)^{2} \\
& \left.+\left(M-E_{n \kappa}^{\mathrm{ps}}+C_{\mathrm{ps}}\right) \beta_{r}\left(\frac{1+e^{-\delta r}}{1-e^{-\delta r}}\right)+\left(M-E_{n \kappa}^{\mathrm{ps}}+C_{\mathrm{ps}}\right) \gamma_{r}\right\} G_{n, \kappa}^{\mathrm{ps}}(r)=0 .
\end{aligned}
$$

It is well known that the above equation cannot be solved exactly due to the centrifugal term $r^{-2}$. In order to get rid of the centrifugal term, we make use of the Greene-Aldrich [37] conventional approximation

$$
\frac{1}{r} \approx \delta \frac{e^{-\delta r}}{\left(1-e^{-\delta r}\right)}
$$

Substituting Eq. (32) into Eq. (31) and applying the transformation, $s=e^{-\delta r}$ yields

$$
\frac{d^{2} G_{n, \kappa}^{\mathrm{ps}}}{d s^{2}}+\frac{1}{s} \frac{d G_{n, \kappa}^{\mathrm{ps}}}{d s}+\frac{1}{s^{2}(1-s)^{2}}\left[-L^{\mathrm{ps}} s^{2}+K^{\mathrm{ps}} s-Q^{\mathrm{ps}}\right] G_{n, \kappa}^{\mathrm{ps}}=0,
$$

where,

$$
\begin{aligned}
L^{\mathrm{ps}} & =\eta_{\kappa}\left(\eta_{\kappa}-1\right)+\left(\frac{2 \kappa}{\delta}+\frac{2 H_{c}}{\delta}+\frac{V_{\mathrm{H}}}{\delta^{2}}\right) V_{\mathrm{H}}-\frac{M \alpha_{r}}{\delta^{2}}+\frac{E_{n \kappa}^{\mathrm{ps}} \alpha_{r}}{\delta^{2}}-\frac{C_{\mathrm{ps}} \alpha_{r}}{\delta^{2}}+\frac{M \beta_{r}}{\delta^{2}}-\frac{E_{n \kappa}^{\mathrm{ps}} \beta_{r}}{\delta^{2}}+\frac{C_{\mathrm{ps}} \beta_{r}}{\delta^{2}} \\
& -\frac{M \gamma_{r}}{\delta^{2}}+\frac{E_{n \kappa}^{\mathrm{ps}} \gamma_{r}}{\delta^{2}}-\frac{C_{\mathrm{ps}} \gamma_{r}}{\delta^{2}}+\frac{M^{2}}{\delta^{2}}-\frac{\left(E_{n \kappa}^{\mathrm{ps}}\right)^{2}}{\delta^{2}}+\frac{M C_{\mathrm{ps}}}{\delta^{2}}+\frac{C_{\mathrm{ps}} E_{n \kappa}^{\mathrm{ps}}}{\delta^{2}}, \\
K^{\mathrm{ps}} & =\frac{V_{\mathrm{H}}}{\delta}+\frac{2 M^{2}}{\delta^{2}}-\frac{2\left(E_{n \kappa}^{\mathrm{ps}}\right)^{2}}{\delta^{2}}+\frac{2 M C_{\mathrm{ps}}}{\delta^{2}}+\frac{2 C_{\mathrm{ps}} E_{n \kappa}^{\mathrm{ps}}}{\delta^{2}}+\frac{2 M \alpha_{r}}{\delta^{2}}-\frac{2 E_{n \kappa}^{\mathrm{ps}} \alpha_{r}}{\delta^{2}}+\frac{2 C_{\mathrm{ps}} \alpha_{r}}{\delta^{2}}-\frac{2 M \gamma_{r}}{\delta^{2}} \\
& +\frac{2 E_{n \kappa}^{\mathrm{ps}} \gamma_{r}}{\delta^{2}}-\frac{2 C_{\mathrm{ps}} \gamma_{r}}{\delta^{2}} \\
Q^{\mathrm{ps}} & =\frac{M^{2}}{\delta^{2}}-\frac{\left(E_{n \kappa}^{\mathrm{ps}}\right)^{2}}{\delta^{2}}+\frac{M C_{\mathrm{ps}}}{\delta^{2}}+\frac{C_{\mathrm{ps}} E_{n \kappa}^{\mathrm{ps}}}{\delta^{2}}-\frac{M \alpha_{r}}{\delta^{2}}+\frac{E_{n \kappa}^{\mathrm{ps}} \alpha_{r}}{\delta^{2}}-\frac{C_{\mathrm{ps}} \alpha_{r}}{\delta^{2}}-\frac{M \beta_{r}}{\delta^{2}}+\frac{E_{n \kappa}^{\mathrm{ps}} \beta_{r}}{\delta^{2}}-\frac{C_{\mathrm{ps}} \beta_{r}}{\delta^{2}} \\
& -\frac{M \gamma_{r}}{\delta^{2}}+\frac{E_{n \kappa}^{\mathrm{ps}} \gamma_{r}}{\delta^{2}}-\frac{C_{\mathrm{ps}} \gamma_{r}}{\delta^{2}},
\end{aligned}
$$

where $\eta_{\kappa}=\kappa+H_{c}$.

\section{B. Spin symmetry in the Dirac equation with Coulomb-Hulthén tensor interaction}

In the spin symmetry limit case, we use the following scalar, vector and tensor potentials:

$$
\begin{aligned}
\Sigma(r)=\alpha_{r} \frac{\cosh ^{2}\left(\frac{r}{2 b}\right)}{\sinh ^{2}\left(\frac{r}{2 b}\right)}+\beta_{r} \frac{\cosh \left(\frac{r}{2 b}\right)}{\sinh \left(\frac{r}{2 b}\right)}+\gamma_{r} & =\alpha_{r}\left(\frac{1+e^{-\delta r}}{1-e^{-\delta r}}\right)^{2}+\beta_{r}\left(\frac{1+e^{-\delta r}}{1-e^{-\delta r}}\right)+\gamma_{r}, \quad \Delta(r)=C_{\mathrm{s}}, \\
U(r) & =-\left(\frac{H_{c}}{r}+V_{\mathrm{H}} \frac{e^{-\delta r}}{1-e^{-\delta r}}\right)
\end{aligned}
$$

Substituting Eqs. (37-38) into Eq. (26) yields

$$
\begin{aligned}
& \left\{\frac{d^{2}}{d r^{2}}-\frac{\kappa(\kappa+1)}{r^{2}}-\frac{2 \kappa H_{c}}{r^{2}}-\frac{2 \kappa V_{\mathrm{H}} e^{-\delta r}}{r\left(1-e^{-\delta r}\right)}-\frac{H_{c}}{r^{2}}-\frac{V_{\mathrm{H}} \delta e^{-\delta r}}{\left(1-e^{-\delta r}\right)^{2}}-\frac{H_{c}^{2}}{r^{2}}-\frac{2 H_{c} V_{\mathrm{H}} e^{-\delta r}}{r\left(1-e^{-\delta r}\right)}\right. \\
& -\frac{V_{\mathrm{H}}^{2} e^{-2 \delta r}}{\left(1-e^{-\delta r}\right)^{2}}-\left(M-E_{n, k}^{s}\right)\left(M+E_{n, k}^{s}-C_{\mathrm{s}}\right)-\left(M+E_{n \kappa}^{\mathrm{ps}}-C_{\mathrm{ps}}\right) \alpha_{r}\left(\frac{1+e^{-\delta r}}{1-e^{-\delta r}}\right)^{2} \\
& \left.-\left(M+E_{n \kappa}^{\mathrm{ps}}-C_{\mathrm{ps}}\right) \beta_{r}\left(\frac{1+e^{-\delta r}}{1-e^{-\delta r}}\right)-\left(M+E_{n \kappa}^{\mathrm{ps}}-C_{\mathrm{ps}}\right) \gamma_{r}\right\} F_{n, k}^{s}(r)=0
\end{aligned}
$$


using the approximation of Eq. (32) for the centrifugal term in Eq. (39), then Eq. (39) leads to the following second order differential equation in view of the change of a variable similar with that in the previous section

$$
\frac{d^{2} F_{n, k}^{s}}{d s^{2}}+\frac{1}{s} \frac{d F_{n, k}^{s}}{d s}+\frac{1}{s^{2}(1-s)^{2}}\left[-\rho^{s} s^{2}+\varsigma^{s} s-f^{s}\right] F_{n, k}^{s}(s)=0,
$$

where

$$
\begin{aligned}
\rho^{s}= & \frac{M^{2}}{\delta^{2}}-\frac{\left(E_{n \kappa}^{s}\right)^{2}}{\delta^{2}}-\frac{M C_{\mathrm{s}}}{\delta^{2}}+\frac{E_{n \kappa}^{s} C_{\mathrm{s}}}{\delta^{2}}+\frac{M \alpha_{r}}{\delta^{2}}+\frac{E_{n \kappa}^{s} \alpha_{r}}{\delta^{2}}-\frac{C_{\mathrm{s}} \alpha_{r}}{\delta^{2}}+\frac{M \gamma_{r}}{\delta^{2}}+\frac{E_{n \kappa}^{s} \gamma_{r}}{\delta^{2}}-\frac{C_{\mathrm{s}} \gamma_{r}}{\delta^{2}}-\frac{M \beta_{r}}{\delta^{2}} \\
- & \frac{E_{n \kappa}^{s} \beta_{r}}{\delta^{2}}+\frac{C_{\mathrm{s}} \beta_{r}}{\delta^{2}}+\Lambda_{\kappa}\left(\Lambda_{\kappa}-1\right)+\left(\frac{2 \kappa}{\delta}+\frac{2 H_{c}}{\delta}+\frac{V_{\mathrm{H}}}{\delta^{2}}\right) V_{\mathrm{H}} \\
\varsigma^{s} & =-\frac{V_{\mathrm{H}}}{\delta}+\frac{2 M^{2}}{\delta^{2}}-\frac{2\left(E_{n \kappa}^{s}\right)^{2}}{\delta^{2}}-\frac{2 M C_{\mathrm{s}}}{\delta^{2}}+\frac{2 E_{n \kappa}^{s} C_{\mathrm{s}}}{\delta^{2}}-\frac{2 M \alpha_{r}}{\delta^{2}}-\frac{2 E_{n \kappa}^{s} \alpha_{r}}{\delta^{2}}+\frac{2 C_{\mathrm{s}} \alpha_{r}}{\delta^{2}}+\frac{2 M \gamma_{r}}{\delta^{2}} \\
& +\frac{2 E_{n \kappa}^{s} \gamma_{r}}{\delta^{2}}-\frac{2 C_{\mathrm{s}} \gamma_{r}}{\delta^{2}} \\
f^{s} & =\frac{M^{2}}{\delta^{2}}-\frac{\left(E_{n \kappa}^{s}\right)^{2}}{\delta^{2}}-\frac{M C_{\mathrm{s}}}{\delta^{2}}+\frac{E_{n \kappa}^{s} C_{\mathrm{s}}}{\delta^{2}}+\frac{M \alpha_{r}}{\delta^{2}}+\frac{E_{n \kappa}^{s} \alpha_{r}}{\delta^{2}}-\frac{C_{\mathrm{s}} \alpha_{r}}{\delta^{2}}+\frac{M \gamma_{r}}{\delta^{2}}+\frac{E_{n \kappa}^{s} \gamma_{r}}{\delta^{2}}-\frac{C_{\mathrm{s}} \gamma_{r}}{\delta^{2}} \\
& +\frac{M \beta_{r}}{\delta^{2}}+\frac{E_{n \kappa}^{s} \beta_{r}}{\delta^{2}}-\frac{C_{\mathrm{s}} \beta_{r}}{\delta^{2}} .
\end{aligned}
$$

where $\Lambda_{\kappa}=\kappa+H_{c}+1$.

\section{Pseudospin and spin symmetry solutions with Coulomb interaction}

In this section, we will solve the solutions of Eqs. (33) and (40) by using the parametric generalization of the NU method [20,32].

\section{Pseudospin symmetry solution with Coulomb interaction}

Now comparing Eq. (2) with Eq. (34),we obtain

$$
\begin{aligned}
& c_{1}=1, \quad c_{2}=1, \quad c_{3}=1, \\
& \xi_{1}=L^{\mathrm{ps}}, \quad \xi_{2}=K^{\mathrm{ps}}, \quad \xi_{3}=Q^{\mathrm{ps}}
\end{aligned}
$$

Other parameters can be obtained from Eq. (5) as

$$
\begin{aligned}
& c_{4}=0, \quad c_{5}=-\frac{1}{2}, \quad c_{6}=\frac{1}{4}+L^{\mathrm{ps}}, \quad c_{7}=-K^{\mathrm{ps}}, \quad c_{8}=Q^{\mathrm{ps}}, \quad c_{9}=\frac{1}{4}+L^{\mathrm{ps}}+Q^{\mathrm{ps}}-K^{\mathrm{ps}}, \\
& c_{10}=1+2 \sqrt{Q^{\mathrm{ps}}}, \quad c_{11}=2+2\left[\sqrt{\frac{1}{4}+L^{\mathrm{ps}}+Q^{\mathrm{ps}}-K^{\mathrm{ps}}}+\sqrt{Q^{\mathrm{ps}}}\right], \\
& c_{12}=\sqrt{Q^{\mathrm{ps}}}, \quad c_{13}=-\frac{1}{2}-\left(\sqrt{\frac{1}{4}+L^{\mathrm{ps}}+Q^{\mathrm{ps}}-K^{\mathrm{ps}}}+\sqrt{Q^{\mathrm{ps}}}\right)
\end{aligned}
$$

Substituting Eqs. (44) and (45) into Eq.(10) yields

$$
\begin{aligned}
& n^{2}+\left(n+\frac{1}{2}\right)+(2 n+1)\left[\sqrt{\frac{1}{4}+L^{\mathrm{ps}}+Q^{\mathrm{ps}}-K^{\mathrm{ps}}}+\sqrt{Q^{\mathrm{ps}}}\right] \\
& -K^{\mathrm{ps}}+2 Q^{\mathrm{ps}}+2 \sqrt{Q^{\mathrm{ps}}\left(\frac{1}{4}+L^{\mathrm{ps}}+Q^{\mathrm{ps}}-K^{\mathrm{ps}}\right)}=0 .
\end{aligned}
$$

From Eqs. (14) and (15) the lower component of the wave functions are abtained as,

$$
G_{n, \kappa}^{\mathrm{ps}}(r)=N_{n, \kappa}^{\mathrm{ps}} e^{-\delta \sqrt{Q^{\mathrm{ps}}}}\left(1-e^{-\delta r}\right)^{\frac{1}{2}+\sqrt{\frac{1}{4}+L^{\mathrm{ps}}+Q^{\mathrm{ps}}-K^{\mathrm{ps}}}} P_{n}^{\left(2 \sqrt{Q^{\mathrm{ps}}}, 2 \sqrt{\frac{1}{4}+L^{\mathrm{ps}}+Q^{\mathrm{ps}}-K^{\mathrm{ps}}}\right)}\left(1-2 e^{-\delta r}\right)
$$


and the other component of the wave function can be obtained as,

$$
F_{n, \kappa}^{\mathrm{ps}}(r)=\frac{1}{M-E_{n, \kappa}^{\mathrm{ps}}+C_{\mathrm{ps}}}\left(\frac{d}{d r}-\frac{\kappa}{r}-\left(\frac{H_{c}}{r}+V_{\mathrm{H}} \frac{e^{-\delta r}}{1-e^{-\delta r}}\right)\right) G_{n, \kappa}^{\mathrm{ps}}(r),
$$

where $N_{n, \kappa}^{\mathrm{ps}}$ is the normalization constant and $E_{n, \kappa}^{\mathrm{ps}} \neq M+C_{\mathrm{ps}}$

\section{Spin symmetry solution}

Again comparing Eqs. (40) and (2) we obtained

$$
\begin{array}{lll}
c_{1}=1, & c_{2}=1, & c_{3}=1, \\
\xi_{1}=\rho^{s}, & \xi_{2}=\varsigma^{s}, & \xi_{3}=f^{s}
\end{array}
$$

From Eq. (5) other parameters are obtained as follows:

$$
\begin{aligned}
& c_{4}=0, \quad c_{5}=-\frac{1}{2}, \quad c_{6}=\frac{1}{4}+\rho^{s}, \quad c_{7}=-\varsigma^{s}, \\
& c_{8}=f^{s}, \quad c_{9}=\frac{1}{4}+\rho^{s}+f^{s}-\varsigma^{s}, c_{10}=1+2 \sqrt{f^{s}} \\
& c_{11}=2+2\left[\sqrt{\frac{1}{4}+\rho^{s}+f^{s}-\varsigma^{s}}+\sqrt{f^{s}}\right], \quad c_{12}=\sqrt{f^{s}} \\
& c_{13}=-\frac{1}{2}-\left[\sqrt{\frac{1}{4}+\rho^{s}+f^{s}-\varsigma^{s}}+\sqrt{f^{s}}\right] .
\end{aligned}
$$

Substituting Eqs. (49) and (50) into Eq. (10) gives the energy eigenvalues as

$$
\begin{aligned}
& n^{2}+n+\frac{1}{2}+(2 n+1)\left(\sqrt{\frac{1}{4}+\rho^{s}+f^{s}-\varsigma^{s}}+\sqrt{f^{s}}\right) \\
& -\varsigma+2 f^{s}+2 \sqrt{f^{s}\left(\frac{1}{4}+\rho^{s}+f^{s}-\varsigma^{s}\right)}=0
\end{aligned}
$$

Finally, we obtain the upper and lower wave function as

$$
\begin{aligned}
F_{n, \kappa}^{s}(r) & =N_{n, \kappa}^{s} e^{-\sqrt{f^{s}} \delta r}\left(1-e^{-\delta r}\right)^{\frac{1}{2}+\sqrt{\frac{1}{4}+\rho^{s}+f^{s}-\varsigma^{s}}} \\
& \times P_{n}^{\left(2 \sqrt{f^{s}}, 2 \sqrt{\frac{1}{4}+\rho^{s}+f^{s}-\varsigma^{s}}\right)}\left(1-2 e^{-\delta r}\right) \\
G_{n, \kappa}^{s}(r)= & \frac{1}{M+E_{n, \kappa}^{s}-C_{\mathrm{s}}} \\
& \times\left(\frac{d}{d r}+\frac{\kappa}{r}+\left(\frac{H_{c}}{r}+V_{\mathrm{H}} \frac{e^{-\delta r}}{1-e^{-\delta r}}\right)\right) F_{n, \kappa}^{s}(r)
\end{aligned}
$$

\section{NUMERICAL RESULTS}

We have obtained the energy eigenvalues and corresponding lower and upper wave functions in terms of the Jacobi polynomials. Moreover, the results obtained in this work are comparable with the previous work. If we set $\alpha_{r}=H_{c}=0$, we can exactly obtain the results of the solution of the Dirac equation under the ManningRosen potential with considering value of $C=0$ [38]. In Tables 1 and 2, we have shown the effect of tensor interactions on energy eigenvalues under pseudospin and spin symmetry limits. Fig. 1 shows energy versus $\delta$ for pseudospin and spin symmetry limits. Figures 2, 3 and 4 represent energy versus potential parameters of $\alpha_{r}, \beta_{r}$ and $\gamma_{r}$ for pseudospin and spin symmetry limits, respectively. Figs. 5 represent the energy for different the values of $C_{\mathrm{ps}}$ and $C_{\mathrm{s}}$ for both symmetry limits.

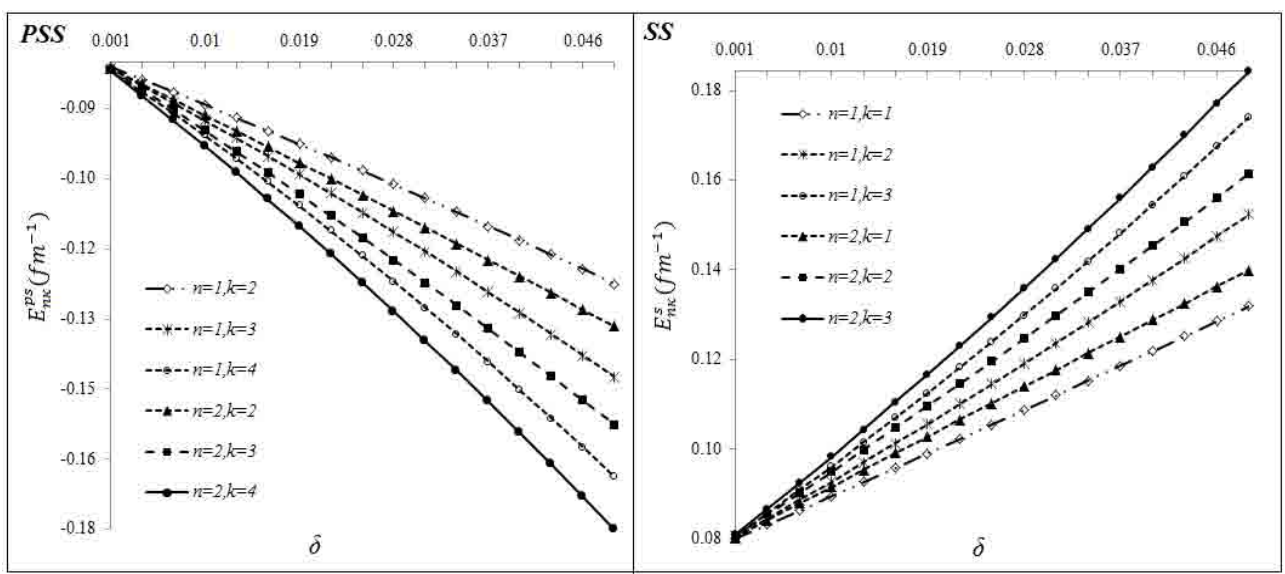

Fig. 1. PSS: Energy vs. $\delta$ for the pseudospin symmetry limit for $V_{\mathrm{H}}=0.5, H_{c}=0.5, M=5 \mathrm{fm}^{-1}, \alpha_{r}=0.6, \beta_{r}=0.5$, $\gamma_{r}=0.4, C_{\mathrm{ps}}=-5$. SS: Energy vs. $\delta$ for the spin symmetry limit for $V_{\mathrm{H}}=0.5, H_{c}=0.5, M=5 \mathrm{fm}^{-1}, \alpha_{r}=-0.6, \beta_{r}=-0.5$, $\gamma_{r}=-0.4, C_{\mathrm{s}}=5$. 


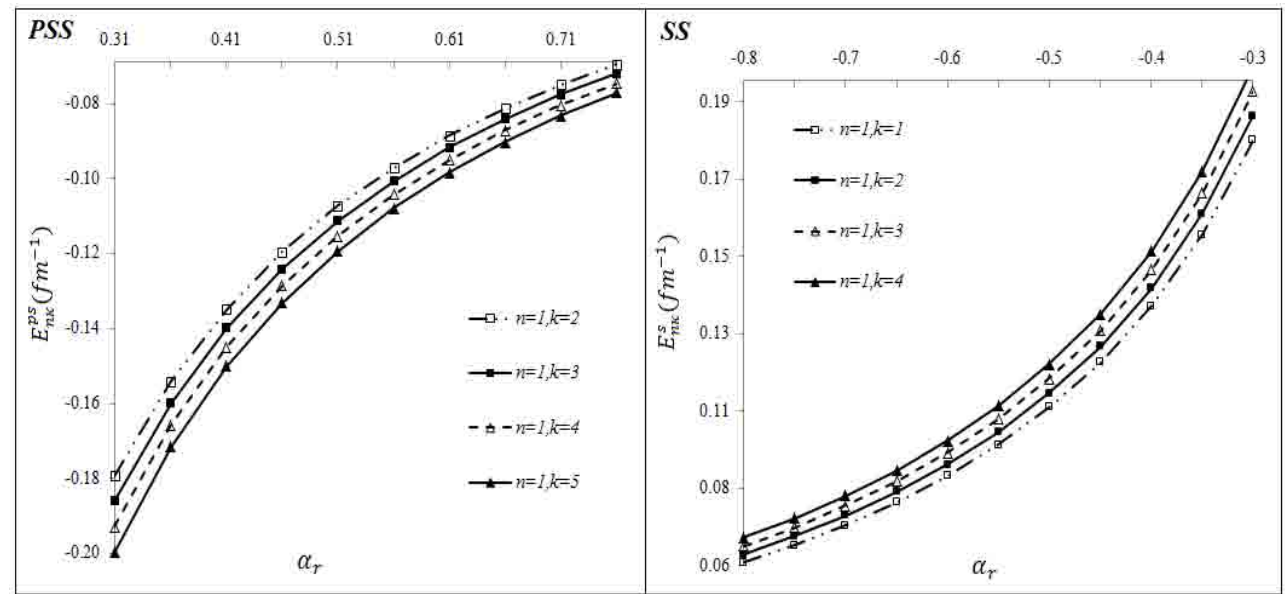

Fig. 2. PSS: Energy vs. $\alpha_{r}$ for the pseudospin symmetry limit for $V_{\mathrm{H}}=0.5, H_{c}=0.5, \delta=0.01, M=5 \mathrm{fm}^{-1}, \beta_{r}=0.5$, $\gamma_{r}=0.4, C_{\mathrm{ps}}=-5 . S S$ : Energy vs. $\alpha_{r}$ for spin symmetry limit for $V_{\mathrm{H}}=0.5, H_{c}=0.5, \delta=0.01, M=5 \mathrm{fm}^{-1}, \beta_{r}=-0.5$, $\gamma_{r}=-0.4, C_{\mathrm{s}}=5$.

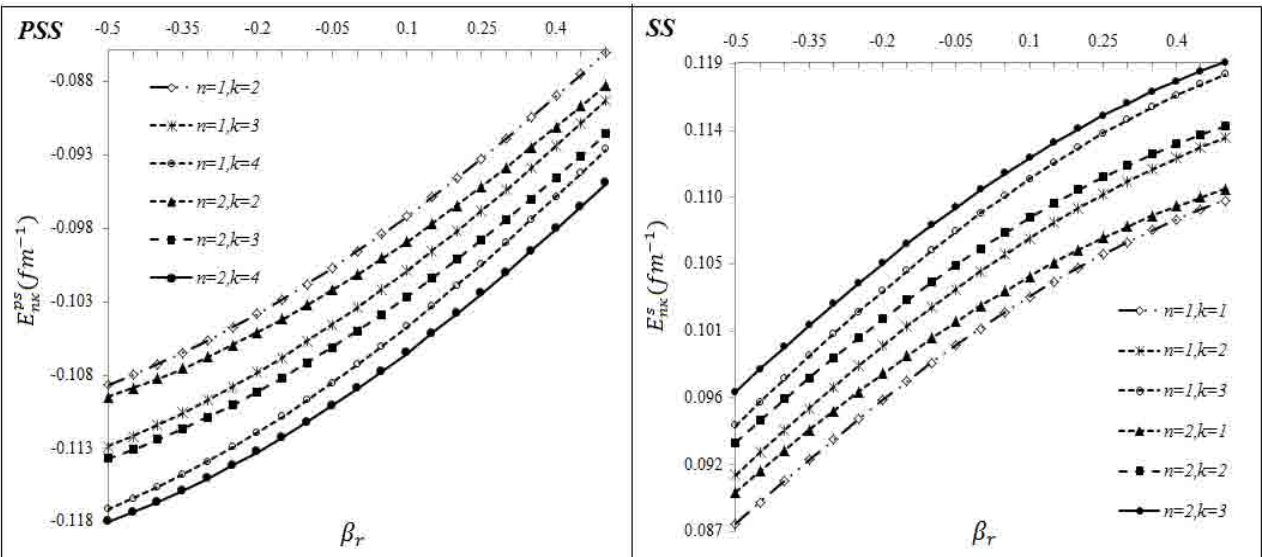

Fig. 3. PSS: Energy vs. $\beta_{r}$ for the pseudospin symmetry limit for $V_{\mathrm{H}}=0.5, H_{c}=0.5, \delta=0.01, M=5 \mathrm{fm}^{-1}, \alpha_{r}=0.6$, $\gamma_{r}=0.4, C_{\mathrm{ps}}=-5$. SS: Energy vs. $\beta_{r}$ for the spin symmetry limit for $V_{\mathrm{H}}=0.5, H_{c}=0.5, \delta=0.01, M=5 \mathrm{fm}^{-1}, \alpha_{r}=-0.6$, $\gamma_{r}=-0.4, C_{\mathrm{s}}=5$.

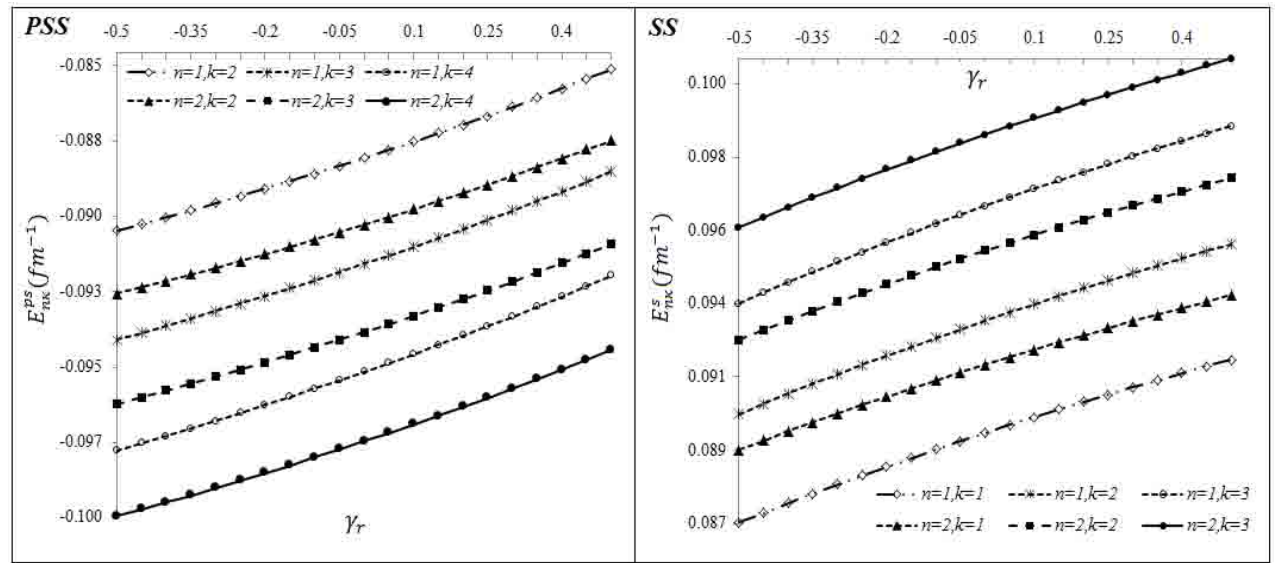

Fig. 4. PSS: Energy vs. $\gamma_{r}$ for the pseudospin symmetry limit for $V_{\mathrm{H}}=0.5, H_{c}=0.5, \delta=0.01, M=5 \mathrm{fm}^{-1}, \alpha_{r}=0.6$, $\beta_{r}=0.5, C_{\mathrm{ps}}=-5$. SS: Energy vs. $\gamma_{r}$ for the spin symmetry limit for $V_{\mathrm{H}}=0.5, H_{c}=0.5, \delta=0.01, M=5 \mathrm{fm}^{-1}, \alpha_{r}=-0.6$, $\beta_{r}=-0.5, C_{\mathrm{s}}=5$. 


\begin{tabular}{|l|l|l||l|l|l|l|}
\hline$\tilde{\ell}$ & $n, \kappa$ & $(\ell, j)$ & $\begin{array}{l}E_{n \kappa}^{\mathrm{ps}}\left(\mathrm{fm}^{-1}\right) \\
V_{\mathrm{H}}=0.5\end{array}$ & $\begin{array}{l}E_{n \kappa}^{\mathrm{ps}}\left(\mathrm{fm}^{-1}\right) \\
V_{\mathrm{H}}=0.5\end{array}$ & $\begin{array}{l}E_{n \kappa}^{\mathrm{ps}}\left(\mathrm{fm}^{-1}\right) \\
V_{\mathrm{H}}=1\end{array}$ & $E_{n \kappa}^{\mathrm{ps}}\left(\mathrm{fm}^{-1}\right)$ \\
$V_{\mathrm{H}}=0.5$ \\
\hline 1 & 1,2 & $0 d_{\frac{3}{2}}$ & -0.087755186 & -0.086121054 & -0.317143611 & -0.08450185 \\
\hline 2 & 1,3 & $0 f_{\frac{5}{2}}$ & -0.09106819 & -0.089404235 & -0.323220125 & -0.087755186 \\
\hline 3 & 1,4 & $0 g_{\frac{7}{2}}$ & -0.094440765 & -0.092747037 & -0.329348801 & -0.09106819 \\
\hline 4 & 1,5 & $0 h_{\frac{9}{2}}$ & -0.097872814 & -0.096149362 & -0.335529423 & -0.094440765 \\
\hline 1 & 2,2 & $1 d_{\frac{3}{2}}$ & -0.090014085 & -0.088356754 & -0.321749995 & -0.086714365 \\
\hline 2 & 2,3 & $1 d_{\frac{3}{2}}$ & -0.093373527 & -0.091686347 & -0.327874664 & -0.090014085 \\
\hline 3 & 2,4 & $1 g_{\frac{7}{2}}$ & -0.096792593 & -0.095075613 & -0.334051527 & -0.093373527 \\
\hline 4 & 2,5 & $1 h_{\frac{9}{2}}$ & -0.100271184 & -0.098524454 & -0.340280369 & -0.096792593 \\
\hline
\end{tabular}

Table 1. Energies in the pseudospin symmetry limit for $\delta=0.01, M=5 \mathrm{fm}^{-1}, \alpha_{r}=0.6, \beta_{r}=0.5, \gamma_{r}=0.4, C_{\mathrm{ps}}=-5$.

\begin{tabular}{|l|l|l||l|l|l|l|}
\hline$\ell$ & $n, \kappa$ & $(\ell, j)$ & $\begin{array}{l}E_{n \kappa}^{s}\left(\mathrm{fm}^{-1}\right) \\
V_{c}=0.5\end{array}$ & $\begin{array}{l}E_{n \kappa}^{s}\left(\mathrm{fm}^{-1}\right) \\
V_{\mathrm{H}}=1 \\
H_{c}=0\end{array}$ & $\begin{array}{l}E_{n \kappa}^{s}\left(\mathrm{fm}^{-1}\right) \\
V_{\mathrm{H}}=0.5\end{array}$ & $\begin{array}{l}E_{n \kappa}^{s}\left(\mathrm{fm}^{-1}\right) \\
V_{\mathrm{H}}=0.5\end{array}$ \\
\hline 1 & 0,1 & $0 P_{\frac{1}{2}}$ & 0.083647822 & 0.315514212 & 0.085244597 & 0.086856283 \\
\hline 2 & 0,2 & $0 d_{\frac{3}{2}}$ & 0.086856283 & 0.321545567 & 0.088482868 & 0.090124340 \\
\hline 3 & 0,3 & $0 f_{\frac{5}{2}}$ & 0.090124340 & 0.327629060 & 0.091780689 & 0.093451901 \\
\hline 4 & 0,4 & $0 g_{\frac{7}{2}}$ & 0.093451901 & 0.333764479 & 0.095137965 & 0.096838869 \\
\hline 1 & 1,1 & $1 P_{\frac{1}{2}}$ & 0.085893701 & 0.320150103 & 0.087514446 & 0.089150100 \\
\hline 2 & 1,2 & $1 d_{\frac{3}{2}}$ & 0.089150100 & 0.326230398 & 0.090800653 & 0.092466093 \\
\hline 3 & 1,3 & $1 f_{\frac{5}{2}}$ & 0.092466093 & 0.332362848 & 0.094146410 & 0.095841590 \\
\hline 4 & 1,4 & $1 g_{\frac{7}{2}}$ & 0.095841590 & 0.338547239 & 0.097551623 & 0.099276497 \\
\hline 1 & 2,1 & $2 P_{\frac{1}{2}}$ & 0.088025193 & 0.324670403 & 0.089669820 & 0.091329359 \\
\hline 2 & 2,2 & $2 d_{\frac{3}{2}}$ & 0.091329359 & 0.330799675 & 0.093003799 & 0.094693129 \\
\hline 3 & 2,3 & $2 f_{\frac{5}{2}}$ & 0.094693129 & 0.336981121 & 0.096397336 & 0.098116410 \\
\hline 4 & 2,4 & $2 g_{\frac{7}{2}}$ & 0.098116410 & 0.343214526 & 0.099850339 & 0.101599111 \\
\hline
\end{tabular}

Table 2. Energies in the spin symmetry limit for $\delta=0.01, M=5 \mathrm{fm}^{-1}, \alpha_{r}=-0.6, \beta_{r}=-0.5, \gamma_{r}=-0.4, C_{\mathrm{s}}=5$.

In Fig. 6, the wave functions are plotted for pseudospin and spin symmetry limits in presence of tensor interaction. It is seen in Fig. 6 that tensor interaction affects only the shape of the wave functions and does not change the node structures of the radial upper and lower components of the Dirac spinors. 


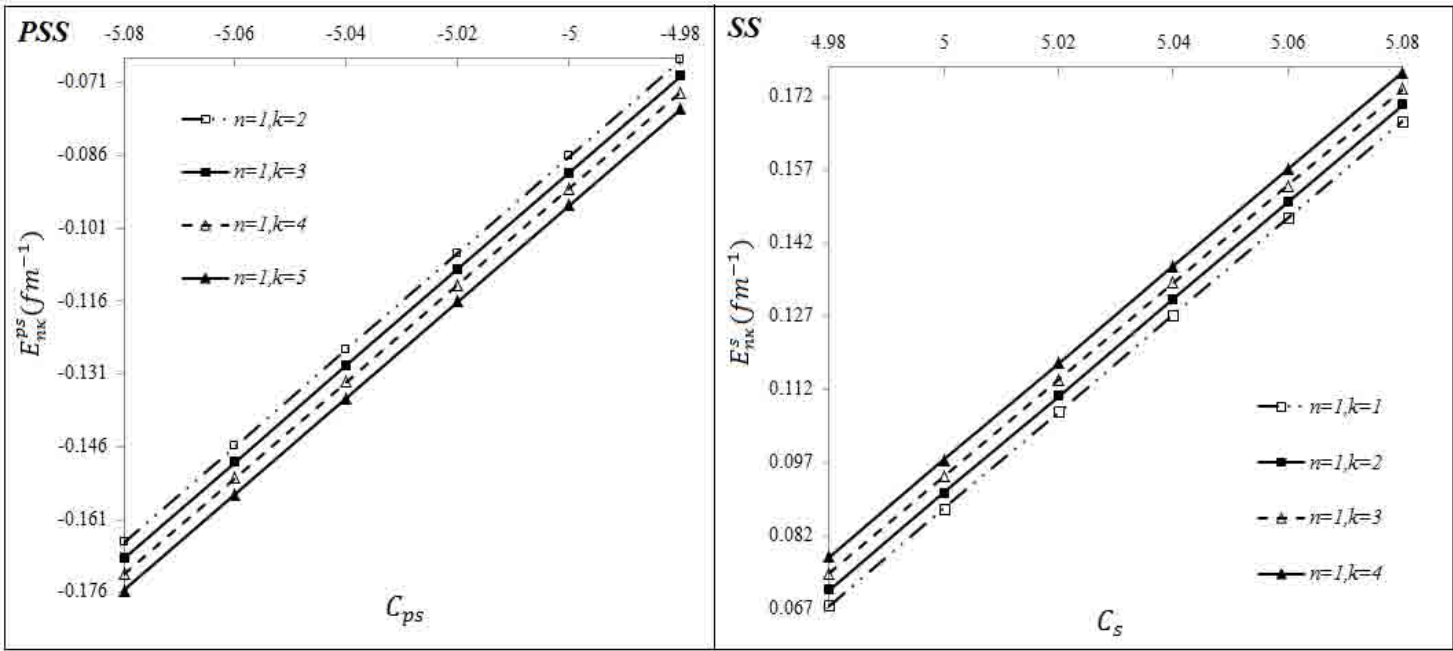

Fig. 5. PSS: Energy vs. $C_{\mathrm{ps}}$ for the pseudospin symmetry limit for $V_{\mathrm{H}}=0.5, H_{c}=0.5, \delta=0.01, M=5 \mathrm{fm}^{-1}, \alpha_{r}=0.6$, $\beta_{r}=0.5, \gamma_{r}=0.4$. SS: Energy vs. $C_{\mathrm{s}}$ for the spin symmetry limit for $V_{\mathrm{H}}=0.5, H_{c}=0.5, \delta=0.01, M=5 \mathrm{fm}^{-1}, \alpha_{r}=-0.6$, $\beta_{r}=-0.5, \gamma_{r}=-0.4$.

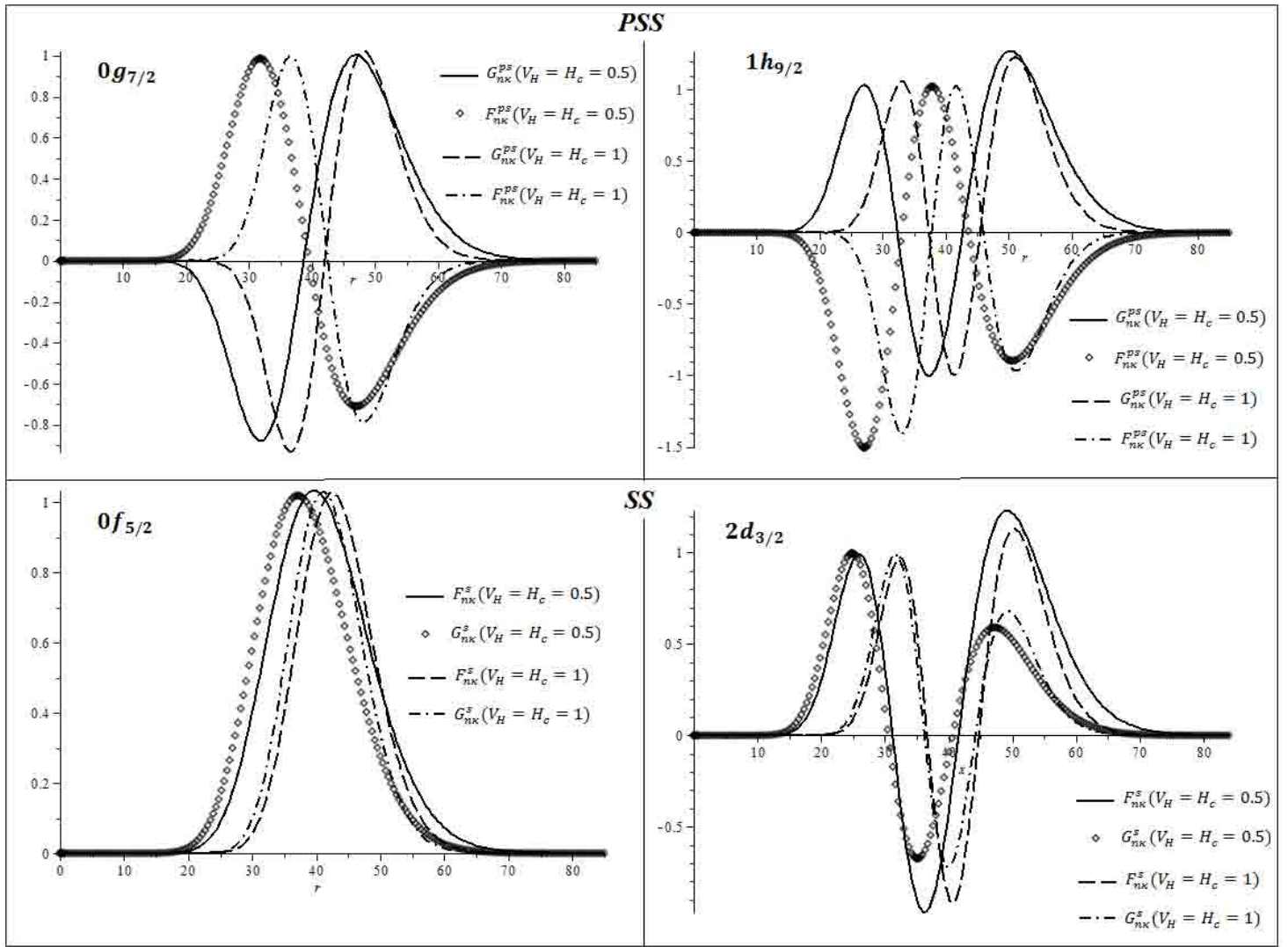

Fig. 6. PSS: Wavefunction of the pseudospin symmetry limit for $\delta=0.01, M=5 \mathrm{fm}^{-1}, \alpha_{r}=0.6, \beta_{r}=0.5, \gamma_{r}=0.4$, $C_{\mathrm{ps}}=-5$. SS: Wavefunction of the spin symmetry limit for $\delta=0.01, M=5 \mathrm{fm}^{-1}, \alpha_{r}=-0.6, \beta_{r}=-0.5, \gamma_{r}=-0.4, C_{\mathrm{s}}=5$. 


\section{CONCLUSIONS}

In this paper,we investigated the Dirac equation with a hyperbolic Manning-Rosen potential under spin and pseudospin symmetry limits. We have calculated the expressions for the energy eigenvalues and unnormalized wave functions by using the parametric NikiforovUvarov method. The motivation of the present work was to investigate the spin and pseudospin symmetry limits of the relativistic Dirac equation which play significant roles in Hadron and nuclear spectroscopy. We obtained the approximate bound states of the Dirac particles for the hyperbolic potential in the presence of spin and pseudospin symmetries including combined Coulomb-like and Hulthen-like tensor interactions using the parametric generalization of the NU method. Our results is a general case of other potential models [39-40].
[1] M. F. Manning, N. Rosen, Phys. Rev. 44, 953 (1933).

[2] N. Rosen, R. M. Morse, Phys. Rev. 42, 210 (1932).

[3] P. Boonserm, M. Visser, preprint arXiv:1005.4483 (2010).

[4] F. Taskın, Int. J. Theor. Phys. 48, 1142 (2009).

[5] G. Wei, S. H. Dong, Phys. Lett. B 686, 288 (2010).

[6] S. M. Ikhdair, R. Sever, preprint arXiv:0801.4271 (2008).

[7] T. Chen, J. Liu, C. Jia, Phys. Scr. 79055002 (2009).

[8] J. N. Ginocchio, Phys. Rev. C 69, 034318 (2004).

[9] J. N. Ginocchio, Phys. Rev. Lett. 78, 436 (1997).

[10] J. N. Ginocchio, Phys. Rep. 414, 165 (2005).

[11] P. R. Page, T. Goldman, J. N. Ginocchio, Phys. Rev. Lett. 66, 204 (2001).

[12] M. K. Bahar, F. Yasuk, Chin. Phys. B 22, 010301 (2012).

[13] E. Maghsoodi, H. Hassanabadi, S. Zarrinkamar, H. Rahimov, Phys. Scr. 85, 055007 (2012).

[14] F. Taskin, Int. J. Theor. Phys. 48, 1142 (2000).

[15] C. S. Jia, P. Guo, X. L. Peng, J. Phys. A 39, 7737 (2006).

[16] J. N. Ginocchio, Phys. Rev. Lett. 95, 252501 (2005).

[17] H. Hassanabadi, E. Maghsoodi, S. Zarrinkamar, H. Rahimov, Chin. Phys. B 21, 120302 (2012).

[18] E. Maghsoodi, H. Hassanabadi, S. Zarrinkamar, FewBody Syst. 53, 535 (2012).

[19] A. N. Ikot, E. Maghsoodi, S. Zarrinkamar, H. Hassanabadi, Few-Body Syst. 54, 2027 (2013).

[20] H. Ciftci, R. L. Hall, N. Saad, J. Phys. A 36, 11807 (2003).

[21] A. F. Nikiforov, V. B. Uvarov, Special Functions of Mathematical Physics (Birkhauser, Basel, 1988).

[22] F. Cooper, A. Khare, U. Sukhatme, Phys. Rep. 251267 (1995).
[23] H. Hassanabadi, S. Zarrinkamar, B. H. Yazarloo, Chin. J. Phys. 50, 788 (2012).

[24] H. Hassanabadi, S. Zarrinkamar, S. Zarrinkamar, H. Rahimov, J. Math. Phys. 53, 022104 (2012).

[25] O. Aydogdu, R. Sever, Few-Body Syst. 47, 193 (2010).

[26] H. Hassanabadi, S. Zarrinkamar, H. Rahimov, Few-Body Syst. 37, 209 (2012).

[27] O. Aydogdu, E. Maghsoodi, H. Hassanabadi, Chin. Phys. B 22, 010302 (2013)

[28] H. Hassanabadi, E. Maghsoodi, S. Zarrinkamar, H. Rahimov, Mod. Phys. Lett. A 26, 2703 (2011).

[29] O. Aydogdu, R. Sever, Eur. Phys. J. A 43, 73 (2010).

[30] H. Hassanabadi, E. Maghsoodi, S. Zarrinkamar, Commun. Theor. Phys. 58, 807 (2012).

[31] A. N. Ikot, H. Hassanabadi, B. H. Yazarloo, S. Zarrinkamar, Int. J. Mod. Phys. E 22, 1350048 (2013).

[32] S. M. Ikhdair, Phys. Scr. 83, 015010 (2011).

[33] C. Tezcan, R. Sver, Int. J. Theor. Phys. 48, 337 (2009).

[34] A. Bharali, Prog. Theor. Exp. Phys. 033 A01 (2013).

[35] S. A. S. Ahmed, L. Buragohain, Electron. J. Theor. Phys. 7, 145 (2010).

[36] A. N. Ikot, E. Maghsoodi, A. D. Antia, S. Zarrinkamar, H. Hassanabadi, Can. J. Phys. 91, 560 (2013).

[37] Y. P. Varshni, Phys. Rev. A 41, 4682 (1990).

[38] R. I. Greene, C. Aldrich, Phys. Rev. A 14, 2363 (1976).

[39] G. F. Wei, S. H. Dong, Phys. Rev. B 686, 288 (2010).

[40] H. Hassanabadi, E. Maghsoodi, S. Zarrinkamar, H. Rahimov, Can. J. Phys. 90, 633 (2012).

[41] H. Hassanabadi, E. Maghsoodi, A. N. Ikot, S. Zarrinkamar, Eur. Phys. J. Plus 128, 79 (2013).

\title{
ГІПЕРБОЛІЧНИЙ ПОТЕНЦІАЛ МЕННІНІА-РОЗЕНА ЗІ СПІНОВОЮ І ПСЕВДОСПІНОВОЮ СИМЕТРІЯМИ РІВНЯННЯ ДІРАКА $З$ ТЕНЗОРНИМИ ВЗАЕМОДІЯМИ ТИПУ КУЛОНА-ГЮЛЬТЕНА
}

\author{
Акпан Н. Ікот ${ }^{1}$, Е. Магсуді ${ }^{2}$, C. Заррінкамар ${ }^{3}$, Г. Гассанабаді ${ }^{2}$ \\ ${ }^{1}$ Університет Порт-Гаркорта, Порт-Гаркорт, Ніґерія \\ ${ }^{2}$ Ісламсъкий університет Азад, Шагруд, Іран \\ ${ }^{3}$ Ісламсъкий університет Азад, Гармсар, Іран
}

\begin{abstract}
Розглянуто рівняння Дірака з гіперболічним потенціалом Меннінга-Розена, який включає комбіновані тензорні взаємодії типу кулона та Гюльтена. За допомогою методу Нікіфорова-Уварова в замкненій формі отримано наближені розв'язки для енергій зв'язаних станів рівняння Дірака з гіперболічним потенціалом Меннінга-Розена та відповідні ненормовані хвильові функції. Наведено результати чисельних розрахунків для демонстрації впливу тензорної взаємодії.
\end{abstract}

